Maneuvering Hydrodynamics of Ellipsoidal Forms

T. Miloh

Hamburg, Technische Universität Hamburg-Harburg, 1977

(C) Technische Universität Hamburg-Harburg

Schriftenreihe Schiffbau

Schwarzenbergstraße 95c

D-21073 Hamburg

http://www.tuhh.de/vss 


\section{INSTITUT FÜR SCHIFFBAU DER UNIVERSITAT HAMBURG}

Maneuvering Hydrodynamics of Ellipsoidal Forms

T. Miloh 
Institut für Schiffbau der Universität Hamburg

MANEUVERING HYDRODYNAMICS OF ELLIPSOIDAL FORMS

by

Touvia Miloh

Dept. of Fluid Mechanics and Heat Transfer

School of Engineering

Tel-Aviv University

Hamburg, Juni 1977

Bericht Nr. 357 


\section{Abstract}

The hydrodynamical forces and moments acting on a tri-axial ellipsoid moving in an incompressible and inviscid fluid are analysed. The rigid ellipsoid is allowed to move in the most general manner with time dependent velocity and six degrees of freedom. The force and moment expressions are obtained by applying the Lagally theorem to the image singularities system representing the body in the presence of exterior disturbances. First expressions for the Lagally force and moment acting on a maneuvering ellipsoid in an unbounded medium are derived and then these expressions are generalized to include the effect of an exterior source moving in an arbitrary manner. It is also shown how the Lagally expressions for an exterior source can be used to obtain closed form expressions for the hydrodynamical forces and moments acting on a maneuvering ellipsoid in the presence of arbitrary exterior disturbance. The analysis which is based on the application of ellipsoidal harmonics is demonstrated in a simple case of propeller-hull interaction. Here the motion of the ellipsoid is restricted to the major axis and the propeller at the stern is represented by an isolated sink in accordance with Dickmann's model. Practical expressions for the thrust deduction coefficient, wake fraction and propeller induced vibration are then derived for ellipsoidal, spheroidal and spherical hulls.

\section{Acknowledgement}

This work has been partially supported by the Sonderforschungsbereich 98

"Schiffstechnik und Schiffbau" at the Institut für Schiffbau, Hamburg. 
1. INTRODUCTION 1

2. MATHEMATICAL BACKGROUND 4

3. THE IMAGE SINGULARITIES SYSTEM OF EXTERIOR SOURCE $\quad 7$

4. THE IMAGE SINGULARITIES SYSTEM OF GENERAL MOTION IN UNBOUNDED MEDIUM

5. HYDRODYNAMICAL FORCES FOR MANEUVERING ELLIPSOID IN INFINITE MEDIUM

6. HYDRODYNAMICAL MOMENTS FOR MANEUVERING ELLIPSOID IN INFINITE MEDIUM

7. HYDRODYNAMICAL FORCES IN DISTURBED OR BOUNDED FLUID DOMAIN

8. HYDRODYNAMICAL MOMENT IN DISTURBED OR BOUNDED FLUID DOMAIN

9. MODEL EXAMPLE: PROPELLER-HULL INTERACTION 26 REFERENCES 


\title{
MANEUVERING HYDRODYNAMICS OF ELLIPSOIDAL FORMS
}

by

\author{
T. Miloh \\ School of Engineering \\ Tel-Aviv University
}

\section{INTRODUCTION}

We are now celebrating the 25 th anniversary of the Institut für Schiffbau. This is an occasion to remember Professor Weinblum, the renowned first director of the Institute. Among the many imprints Professor Weinblum left in the field of ship theory were his studies of Maneuvering Hydrodynamics of general ship forms and of ellipsoidal forms in particular. Professor Weinblum advocated the use of ellipsoidal forms as an approximation for general ship forms and his often repeated statement "the ellipsoid is God's gift to naval architects!" (quoted by Newman (1972)) has stimulated many valuable studies. His approach to ship maneuvering extensively used the Lagally theorem of which Professor Weinblum was one of the main promotors and one of the first to realize its power and versatility. For these reasons the present paper which analyses general maneuvering of ellipsoids by employing the Lagally theorem is dedicated to Professor Weinblum's memory in this jubilee year of the Institut für Schiffbau.

Due to the ever increasing ship size and traffic density there is currently a growing interest in the area of ship maneuvering sspecially in confined and shallow water. In spite of this the contributions from theoretical hydrodynamics to the field of ship maneuvering have been limited as pointed out by the 
Maneuverability Committee of the 14th International Towing Tank Conference held in 1975. Following the recommendation of this conference, it was decided to analyse the problem of a general ellipsoid maneuvering in the proximity of a general disturbance (wall, free-surface, body, propeller, etc.). As is customary in maneuvering studies, the effect of compressibility, viscosity and Froude number will be neglected and the free surface will be replaced by the double-model approximation.

The determination of the hydrodynamical forces and moments acting on a maneuvering ship in a bounded or disturbed fluid medium is indeed a hard task. The problem may be simplified by using a tri-axial allipsoid as an approximation to the ship hull taking advantage of some analytic tools which are particularly tailored for ellipsoided shapes. The idea of replacing a ship hull by an "equivalent ellipsoid" was first suggested by Weinblum (1936) and later used by Eggers (1960), Wu and Chwang (1974), and M1loh and Landweber (1977).

The present paper considers the most general time-dependent motion of a tri-axial ellipsoid with six degrees of freedom in a disturbed fluid medium. The fluid is assumed to be inviscid and incompressible and the effect of fluid separation is ignored. The mathematical formulation employs the classical theory of ellipsoid harmonics (Hobson 1955) together with some recent contributions to potential flow theory (Miloh 1973, 1974). These theories are particularly appealing in view of the recent progress in computerizing the numerical computation of the varfous Lame functions (Walter 1971).

The concept of "force and moment influence functions" is introduced to calculate the forces and moments experienced by a maneuvering ellipsoid in the proximity of an exterior disturbance which may be represented by an arbitrary distribution of singularities. First general expressions for the forces and moments acting on a maneuvering ellipsold in the presence of an exterior 
moving source with a time dependent output are derived. It is then shown how these "force and moment influence functions" may be used to determine the hydrodynamical forces and moments acting on an ellipsoid moving in the proximity of a general prescribed system of singularities representing, wall, free-surface, solid bodies, etc. This concept is somewhat analogous to the use of the Green's function of a unit source to determine the velocity potential of an arbitrary distribution of singularities by formulating an integral equation.

The general expression for the forces and moments acting on an ellipsoid in the proximity of an exterior source are obtained by applying the Lagally theorem to the image singularity system as described in Section 3 for the case of a stationary ellipsoid in the presence of exterior source, and in Section 4 for maneuvering ellipsoid in unbounded medium. The direct application of the Lagally theorem to determine the hydrodynamical forces and moments acting on a maneuvering ellipsoid in unbounded medium is demonstrated in Sections 5 and 6, respectively. Finally, expressions for the forces and moments experienced by an ellipsoid moving with six degrees of freedom in the proximity of an exterior unsteady source with arbitrary spacial motion are derived in Sections 7 and 8 . It is also shown how these "influence functions" may be used to obtain closed-form expressions for the hydrodynamical forces and moments acting on the ellipsoid in the proximity of a general prescribed singularity system representing fluid or solld boundaries.

In Section 9 the general expressions thus derived are demonstrated to calculate the force experienced by the ellipsoid in a relatively simple case, namely an allipsoid translating in the direction of its major axis in the presence of an exterior sink with time dependent output lying on the same axis. The results of this example may be found useful in analysing propeller hull interaction, in accordance with Dickmann's isolated-sink model of the propeller. Other parameters such as potential wake fraction, thrust deduction coefficient and propeller induced vibratory force, are also briefly discussed. 


\section{MATHEMATICAL BACKGROUND}

In order to solve the Laplace equation in a domain bounded (internally or external1y) by a tri-axial ellipsoidal surface, it is convenient to formulate the problem in ellipsoidal coordinates. The ellipsoidal (confocal) coordinates are defined by the solutions of the following cubic equation in $X$;

$$
\frac{x_{1}^{2}}{x^{2}}+\frac{x_{2}^{2}}{x^{2}-h^{2}}+\frac{x_{3}^{2}}{x^{2}-k^{2}}=1
$$

for a fixed value of the Cartesian coordinates $\left(x_{1}, x_{2}, x_{3},\right)$. Both $h$ and $k$ are related to the three semi-axes of the ellipsoid $i_{. e} a_{1}, a_{2}$, and $a_{3}$ in the $x_{1}$, $x_{2}$ and $x_{3}$ directions respectively by

$$
\mathrm{k}^{2}=\mathrm{a}_{1}^{2}-\mathrm{a}_{3}^{2} ; \mathrm{h}^{2}=\mathrm{a}_{1}^{2}-\mathrm{a}_{2}^{2}
$$

such that $a_{1}>a_{2}>a_{3}$

The three roots of (1), here denoted by $\rho, \mu$ and $\nu$, are so chosen that

$$
\infty>\rho^{2} \geq k^{2} ; k^{2} \geq \mu^{2} \geq h^{2} ; h^{2}>\nu^{2} \geq 0
$$

The three surfaces $\rho=$ constant (ellipsoids), $\mu=$ constant (hyperboloids of one sheet) and $\nu=$ constant (hyperboloids of two sheets) form a spacial triply orthogonal coordinate system. In such a coordinate system the canonical ellipsoid with the semi-axes $\left(a_{1}, a_{2}, a_{3}\right)$ is simply given by $\rho=a_{1}$.

The transformation between the ellipsoidal and the Cartesian coordinates is given in Hobson (1955, p. 454) 


$$
\begin{aligned}
& x_{1}^{2}=\frac{\rho^{2} \mu^{2} \nu^{2}}{h^{2} k^{2}} \\
& x_{2}^{2}=\frac{\left(\rho^{2}-h^{2}\right)\left(\mu^{2}-h^{2}\right)\left(h^{2}-\nu^{2}\right)}{h^{2}\left(k^{2}-h^{2}\right)} \\
& x_{3}^{2}=\frac{\left(\rho^{2}-k^{2}\right)\left(k^{2}-\mu^{2}\right)\left(k^{2}-\nu^{2}\right)}{k^{2}\left(k^{2}-h^{2}\right)}
\end{aligned}
$$

The three linearizing factors of the above transformation in the $\rho, \mu$ and $\nu$ directions are here denoted by $\bar{h}_{\rho}, \bar{h}_{\mu}$, and $\bar{h}_{\nu}$ respectively and are given by

$$
\begin{aligned}
& \bar{h}_{\rho}^{2}=\frac{\left(\rho^{2}-\mu^{2}\right)\left(\rho^{2}-\nu^{2}\right)}{\left(\rho^{2}-h^{2}\right)\left(\rho^{2}-k^{2}\right)} \\
& \bar{h}_{\mu}^{2}=\frac{\left(\rho^{2}-\mu^{2}\right)\left(\mu^{2}-\nu^{2}\right)}{\left(\mu^{2}-h^{2}\right)\left(k^{2}-\mu^{2}\right)} \\
& \bar{h}_{\zeta}^{2}=\frac{\left(\rho^{2}-\nu^{2}\right)\left(\mu^{2}-\nu^{2}\right)}{\left(h^{2}-\nu^{2}\right)\left(k^{2}-\nu^{2}\right)}
\end{aligned}
$$

A normal (separable) solution of the Laplace equation at the point $M(\rho, \mu, U)$ which is regular at the origin may be written as

$$
P_{n}^{m}(M)=P_{n}^{m}(\rho, \mu, u)=E_{n}^{m}(\rho) \quad E_{n}^{m}(\mu) \quad E_{n}^{m}(u)
$$

where $E_{n}^{m}$ denotes the Lame function of the first kind of order $n$ and degree $m$. Here both $n$ and $m$ are positive integers satisfying $2 n+1 \geq m \geq 1$. 
Similarly a normal solution of the Laplace equation at $M(\rho, \mu, U)$ which is regular at infinity is

$$
Q_{n}^{m}(M)=Q_{n}^{\mathfrak{m}}(\rho, \mu, U)=F_{n}^{m}(\rho) \quad E_{n}^{m}(\mu) \quad E_{n}^{m}(U)
$$

where $F_{n}^{m}$ denotes the Lame function of the second kind which is related to $\mathrm{E}_{\mathrm{n}}^{\mathrm{m}}$ by

$$
F_{n}^{m}(\rho)=I_{n}^{m}(\rho) E_{n}^{m}(\rho)
$$

where, following Hobson (1955, p. 472)

$$
I_{n}^{m}(\rho)=(2 n+1) \int_{\rho}^{\infty} \frac{d \rho}{\left[E_{n}^{m}(\rho)\right]^{2} \sqrt{\left(\rho^{2}-h^{2}\right)\left(\rho^{2}-k^{2}\right)}}
$$

Both the interior $P_{n}^{m}(M)$ and the exterior $Q_{n}^{m}(M)$ ellipsoidal harmonics are linearly independent and form a complete set (Whittaker and Watson 1927, p. 560).

The orthogonality relations of the Lame functions of the first kind may be expressed in the following form (Miloh 1973)

$$
\int \frac{E_{n}^{m}(\mu) E_{n}^{m}(u) E_{n^{\prime}}^{m^{\prime}}(u) E_{n^{\prime}}^{m^{\prime}}(u)}{S \sqrt{\left(a_{1}^{2}-\mu^{2}\right)\left(a_{1}^{2}-v^{2}\right)}} d S=0
$$

for $n \neq n^{\prime}$ or $m \neq m^{\prime}$ where the intrgration is carried over the surface $S$ of the canonical ellipsoid $\rho=a_{1}$. For the particular case where $n=n^{\prime}$ and $m=m^{\prime}$ we define 


$$
\gamma_{n}^{m}=\gamma_{n}^{m}\left(a_{1}, a_{2}, a_{3}\right)=\int \frac{\left[E_{n}^{m}(\mu) E_{n}^{m}(u)\right]^{2}}{\sqrt{\left(a_{1}^{2}-\mu^{2}\right)\left(a_{1}^{2}-u^{2}\right)}} d s
$$

where it can be shown that in addition to dependence on the three semi-axes $\gamma_{n}^{m}$ is a function of the transcendental number $\pi$.

In solving harmonic boundary value problems involving ellipsoidal surfaces, it is advantageous to have an expression for basic potential function which is the inverse of the distance between the two spacial points $M(\rho, \mu, U)$ and $M^{\prime}\left(\rho^{\prime}, \mu^{\prime}, U^{\prime}\right)$ such that $\rho^{\prime}>\rho$. Such an expression has been recently derived by Miloh (1973) in which the inverse value of the distance $R$ between $M$ and $M^{\prime}$ is given in terms of a double summation of ellipsoidal harmonics,

$$
\frac{1}{R\left(M, M^{\prime}\right)}=\sum_{n=0}^{\infty} \sum_{m=1}^{2 n+1} \frac{4 \pi}{(2 n+1) Y_{n}^{m}} Q_{n}^{m}\left(M^{\prime}\right) P_{n}^{m}(M)
$$

The above relation, which is essential in any formulation of the problem as an integral equation, serves as the basis for the following analysis.

\section{THE IMAGE SINGULARITIES SYSTEM OF EXTERIOR SOURCE}

In this section we derive an expression for the image singularities system over the surface of the ellipsoid which will preserve its shape in the presence of an exterior source. The velocity potential induced at $M^{\prime}$ by a unit sink located at $M$ in an unbounded medium is given by (16). Introduction of an ellipsoidal boundary given by $\rho=a_{1}$ (such that $\rho^{\prime}>a_{1}$ ) into the medium, disturbs the flow and the modified velocity potential of the source $\Phi_{\mathbf{g}}$, may be written as 


$$
\Phi_{s}\left(M, M^{\prime}\right)=-\sum_{n=0}^{\infty} \sum_{m=1}^{2 n+1} \frac{4 \pi}{(2 n+1) \gamma_{n}^{m}} Q_{n}^{m}\left(M^{\prime}\right)\left[P_{n}^{m}(M)-c_{n}^{m} Q_{n}^{m}(M)\right]
$$

where $C_{n}^{m}$ are coefficients to be determined. Applying the boundary conditions of the Neumann type, i.e. that the normal derivative of $\Phi_{s}$ vanishes on $\rho=a_{1}$ yields

$$
C_{n}^{m}=C_{n}^{m}\left(a_{1}\right)=\frac{E_{n}^{m}\left(a_{1}\right)}{\dot{F}_{n}^{m}\left(a_{1}\right)} \quad ; \quad D_{n}^{m}=D_{n}^{m}\left(a_{1}\right)=\frac{C_{n}^{m}}{(2 n+1) \gamma_{n}^{m}}
$$

where the dot denotes differentiation with respect to the argument, and $D_{n}^{m}$ is defined here for later use.

It has been shown that an arbitrary exterior ellipsoidal harmonic may be represented by its "ultimate" image singularities system consisting of a continuous distribution of sources or normal doublets over the "fundamental ellipsoid" given by $\rho=k$ (Miloh 1974). Alternatively an exterior ellipsoidal harmonic may be also represented by its surface image singularities system which consists of surface distribution of sources over the canonical ellipsoid $\rho=a_{1}$ given by (Miloh 1973),

$$
Q_{n}^{m}\left(M^{\prime}\right)=\frac{2 n+1}{4 \pi E_{n}^{m}\left(a_{1}\right)} \int_{S} \frac{E_{n}^{m}(\mu) E_{n}^{m}(U)}{\sqrt{\left(a_{1}^{2}-\mu^{2}\right)\left(a_{1}^{2}-u^{2}\right)}} \frac{d S}{R\left(M_{e}, M^{\prime}\right)}
$$

where $M_{e}$ denotes a point on the canonical ellipsoid 1.e. $M_{e}\left(a_{1}, \mu, U\right)$. Combining (16) and (19) renders the following expressions for the surface source distribution, 


$$
\sigma_{s}\left(M_{e}, M^{\prime}\right)=-\sum_{n=0}^{\infty} \sum_{m=1}^{2 n+1} \frac{C_{n}^{m} Q_{n}^{m}\left(M^{\prime}\right)}{\gamma_{n}^{m} E_{n}^{m}\left(a_{1}\right)} \cdot \frac{E_{n}^{m}(\mu) E_{n}^{m}(U)}{\sqrt{\left(a_{1}^{2}-\mu^{2}\right)\left(a_{1}^{2}-u^{2}\right)}}
$$

which is the desired expression for the induced source distribution at $M_{e}\left(a_{1}, \mu, U\right)$ resulting from an exterior source of unit strength lying at $M^{\prime}\left(\rho^{\prime}, \mu^{\prime}, U^{\prime}\right)$.

\section{THE IMAGE SINGULARITIES SYSTEM OF GENERAL MOTION IN UNBOUNDED MEDIUM}

Consider a tri-axial ellipsold moving with six degrees of freedom in an unbounded fluid medium otherwise at rest. The general motion consists of a translatory velocity vector $\vec{v}\left(v_{1}, v_{2}, v_{3}\right)$ where $v_{1}, v_{2}$ and $v_{3}$ denote the velocity components in the $x_{1}, x_{2}$, and $x_{3}$ directions respectively. In addition, the ellipsoid is rotating about its major axes with angular velocity $\vec{\Omega}\left(\Omega_{1}, \Omega_{2}, \Omega_{3}\right)$ where again $\Omega_{1}, \Omega_{2}$ and $\Omega_{3}$ denote the components of the angular velocity vector about $x_{1}$, $x_{2}$ and $x_{3}$ respectively. The six velocity components are assumed to be time dependent for the sake of generality. It is also understood that the Cartesion coordinate system $\left(x_{1}, x_{2}, x_{3}\right)$ is rigidly attached to the ellipsoid.

The solution for the exterior velocity potential $\Phi_{0}$ induced by such a motion at a point $M_{e}$ on the ellipsoid may be found by solving six Neumamn problems (Miloh 1973) which render,

$$
\Phi_{0}\left(M_{e}\right)=\sum_{i}\left[V_{i} \alpha_{i}\left(M_{e}\right)+\Omega_{i} \beta_{i}\left(M_{e}\right)\right] \quad i=1,2,3
$$

The six coefficients $\alpha_{i}\left(M_{e}\right)$ and $\beta_{i}\left(M_{e}\right)$ are given by 


$$
\begin{aligned}
& \alpha_{i}\left(M_{e}\right)=K C_{1}^{i}\left(\left|a_{i+1}^{2}-a_{i+2}^{2}\right|\right)^{1 / 2} Q_{1}^{i}\left(M_{e}\right) ; \\
& \beta_{i}\left(M_{e}\right)=K C_{2}^{6-i} \frac{\left(\left|a_{i+1}^{2}-a_{i+2}^{2}\right|\right)}{a_{i+1}^{2}+a_{i+2}^{2}} Q_{2}^{6-i}\left(M_{e}\right)
\end{aligned}
$$

where

$$
1 / K=\left[\left(a_{1}^{2}-a_{2}^{2}\right)\left(a_{2}^{2}-a_{3}^{2}\right)\left(a_{1}^{2}-a_{3}^{2}\right)\right]^{1 / 2}=h k \sqrt{k^{2}-h^{2}}
$$

and defining $a_{3+i}=a_{i}$. The various Lame functions appearing in (22) are defined by (Hobson 1955, p. 473).

$E_{1}^{1}(\rho)=\rho \quad ; \quad E_{1}^{2}(\rho)=\sqrt{\left(\rho^{2}-h^{2}\right)} ; \quad E_{1}^{3}(\rho)=\sqrt{\left(\rho^{2}-k^{2}\right)}$

$E_{2}^{3}(\rho)=\rho \sqrt{\left(\rho^{2}-h^{2}\right)} ; \quad E_{2}^{4}(\rho)=\rho \sqrt{\left(\rho^{2}-k^{2}\right)} ; \quad E_{2}^{5}(\rho)=\sqrt{\left(\rho^{2}-h^{2}\right)\left(\rho^{2}-k^{2}\right)}$

implying, that

$\mathrm{P}_{1}^{1}(\mathrm{M})=\mathrm{hkx}_{1} \quad ; \quad \mathrm{P}_{1}^{2}(\mathrm{M})=\mathrm{hx_{2 }} \sqrt{\mathrm{k}^{2}-\mathrm{h}^{2}} \quad ; \quad \mathrm{P}_{1}^{3}(\mathrm{M})=k \mathrm{x}_{3} \sqrt{\mathrm{k}^{2}-\mathrm{h}^{2}}$

$\mathrm{P}_{2}^{3}(M)=\mathrm{h}^{2} \mathrm{kx}_{1} \mathrm{x}_{2} \sqrt{\mathrm{k}^{2}-\mathrm{h}^{2}} ; \mathrm{P}_{2}^{4}(M)=\mathrm{hk}^{2} \mathrm{x}_{2} \mathrm{x}_{3} \sqrt{\mathrm{k}^{2}-\mathrm{h}^{2}} ; \mathrm{P}_{2}^{5}(M)=h \mathrm{k}\left(\mathrm{k}^{2}-\mathrm{h}^{2}\right) \mathrm{x}_{2} \mathrm{x}_{3}$

The various exterior harmonics $Q_{n}^{m}$ appearing in (22) are related to the Lame functions of the first kind listed in (23) in the manner given by (11), (12), and (13).

The final expression for the surface image source distribution $\sigma_{0}$ induced by the general motion at points $M_{e}$ is obtained by substituting (19) in (21) which yields 


$$
\sigma_{0}\left(M_{e}\right)=-\frac{1}{\sqrt{\left.a_{1}^{2}-\mu^{2}\right)\left(a_{1}^{2}-v^{2}\right)}} \sum_{i=1}^{3} \frac{3 V_{i} \alpha_{i}\left(M_{e}\right)}{4 \pi a_{i} F_{1}^{i}\left(a_{1}\right)}+\frac{5 a_{i} \Omega_{i} \beta_{i}\left(M_{e}\right)}{3 \downarrow F_{2}{ }^{6-i}\left(a_{1}\right)}
$$

where $\forall$ denotes the volume of the ellipsoid.

\section{HYDRODYNAMICAL FORCES FOR MANEUVERING ELLIPSOID IN INFINITE MEDIUM}

The hydrodynamical forces acting on a maneuvering Rankine body may be calculated by applying the Lagally (1922) theorem, once the Image singularity system which generates the body has been determined. The powerful theorem of Lagally, originally given for steady flow conditions, has been extended by Cummins (1953) and by Landweber and Yih (1956) to cases of unsteady flows.

The desired expression for the Lagally force $\vec{T}\left(T_{1}, T_{2}, T_{3}\right)$ experienced by a Rankine body which is generated by a continuous surface source distribution is given by (Landweber and Yih 1956).

$$
T_{i}=\rho_{F}\left[\forall \frac{d V_{i}}{d t^{\star}}-4 \pi \frac{d}{d t^{\star}} \int_{S} \sigma x_{i} d S-4 \pi \int_{S} \sigma v_{i} d S\right]
$$

where $\rho_{F}$ denotes the mass density of the fluid, $\forall$ is the volume of the body, $\vec{V}_{c}\left(v_{1}, v_{2}, v_{3}\right)$ is the velocity of the body-volume centroid and $\vec{r}\left(x_{1}, x_{2}, x_{3}\right)$ denotes the position radius vector of a point lying on the body surface. The image system of sources of the exterior flow field is here denoted by $\sigma(\vec{r}, t)$ and $\vec{v}\left(v_{1}, v_{2}, v_{3}\right)$ is the velocity vector induced at the location of the singularity due to all external flow producing mechanisms, hence neglecting contributions from 
all internal singularities. Time is represented by $t$ and the symbol $\frac{d}{d t *}$ denotes absolute time derivatives which are related to the relative time derivative $\frac{d}{d t}$ via

$$
\frac{d q_{i}}{d t^{*}}=\frac{d q_{i}}{d t}+e_{i j k} \Omega_{j} q_{k} \quad i, j, k=1,2,3
$$

for some arbitrary vector $\vec{q}\left(q_{1}, q_{2}, q_{3}\right)$ where $e_{i j k}$ denotes the permutation tensor. For the case where the ellipsoid is moving in an unbounded medium otherwise at rest there are no flow-producing mechanisms in the region exterior to the body and therefore $\vec{v}=0$. Substituting this value in (26) and denoting the Lagaliy force for the unbounded case by $\overrightarrow{\mathrm{T}}_{\mathrm{o}}\left(\mathrm{T}_{\mathrm{o}_{1}}, \mathrm{~T}_{\mathrm{o}_{2}}, \mathrm{~T}_{\mathrm{o}_{3}}\right)$, one obtains from (25)

$$
T_{o i}=\rho_{F} \frac{d}{d t *}\left[\left(\forall v_{i}-4 \pi \int_{S} \sigma_{o} x_{i} d S\right)\right]
$$

where $\sigma_{0}(\vec{r}, t)$ is given by $(24)$.

An expression for the components $x_{i}$ of the radius vector is obtained by substituting (22) and (23) in (4), (5) and (6) which yields

$$
x_{i}=\frac{a_{i} a_{i}(M)}{c_{1}^{i} F_{1}^{i}\left(a_{1}\right)}
$$

Introducing (28) into (27) and using the orthogonality conditions of the Lame functions given in (15), renders

$$
\int_{S} \sigma_{0} x_{i} d S=-v_{i} c_{1}^{i}
$$


where in deriving (29) we used the numertcal values of the three coefficients $\gamma_{1}^{i}$

$$
\gamma_{1}^{i}=\frac{4 \pi}{3 k^{2}\left(a_{i+1}^{2}-a_{i+2}^{2}\right)}
$$

as computed by the substitution of (23) into (15) and performing the integration over $\rho=a_{1}$.

The final result for the Lagally force for maneuvering ellipsoid in unbounded medium is obtained after operating with the absolute time derivative on (27) which yields,

$$
T_{o i}=\rho_{F} \forall\left\{\left(1+\frac{3 C_{1}^{i}}{K}\right) \frac{d V_{i}}{d t}-e_{i j k}\left(1+\frac{3 C_{1}^{j}}{k}\right) v_{j k} \Omega_{k}\right\}
$$

The above relation may be further simplified by recalling the Taylor (1928) added mass theorem which renders the following expressions for the three translatory added mass coefficients $A_{i i}$ corresponding to pure translation in the $x_{i}$ directions

$$
\forall\left(1+A_{i i}\right)=4 \pi \int_{S} \sigma_{0} x_{i} d S
$$

which, because of (29), yields

$$
A_{i i}=-\left(1+\frac{4 \pi c_{1}^{i}}{\eta}\right)
$$


Substitution of (33) into (31) enables us to express the latter in the more familiar form

$$
T_{o i}=-\rho_{E} \forall\left[A_{i i} \frac{d V_{i}}{d t}-e_{i j k} A_{j j} V_{j} \Omega_{k}\right]
$$

The above relation is also valid for the more general class of maneuvering bodies with three orthogonal planes of symmetry (Kochin et al., 1964, p. 401) in an undisturbed medium. The classical derivation of (34) is by employing the method of Kirchhoff-Lagrange (Lamb $1932, p .188$ ) which is based on energy conservation. Here we rederive the same relations for ellipsoidal bodies using a different approach, i.e., by applying the Lagally and the Taylor theorems which are based on the momentum conservation principle. It should be also mentioned that for ellipsoidal bodies there exist closed-form expressions for the three translatory added-mass coefficients in terms of the tabulated Legendre incomplete elliptic integrals (Miloh 1973, 1974).

\section{HYDRODYNAMICAL MOMENTS FOR MANEUVERING ELLIPSOID IN INFINITE MEDIUM}

In order to express the Lagally moment experienced by a Rankine body in terms of its image system of singularities it was found convenient to decompose the exterior velocity potential $\Phi$ into two parts; one which is due to the motion of the body alone which is again decomposed into six velocity potentials in the manner of Kirchhoff, and a second part $\Phi_{s}$ which is due to the motion of the external boundaries and other flow-producing mechanisms when the body is at rest. The total exterior velocity potential is then written as

$$
\Phi(M)=\sum_{i=1}^{3}\left[\mathrm{~V}_{1} \phi_{1}(M)+\Omega_{i} \phi_{3+1}(M)\right]+\phi_{s}(M)
$$


where the various velocity potentials in (35) satisfy the following kinematic boundary conditions on the body surface.

$$
\frac{\partial \phi_{i}}{\partial \underset{\sim}{n}}={\underset{\sim}{\mathfrak{n}}}_{i} \quad ; \quad \frac{\partial \phi_{3+j}}{\partial \underset{\sim}{n}}=e_{i j k} x_{j} \underset{\sim k}{p_{k}} \quad ; \quad \frac{\partial \phi_{s}}{\partial \underset{\sim}{n}}=0
$$

Here $\underset{\sim}{\vec{n}}\left(\mathrm{n}_{1},{\underset{\sim}{n}}_{2}, \underset{\sim 3}{\mathrm{n}_{3}}\right)$ denotes the unit normal vector in the outward direction of the surface.

The Lagally expressions for the hydrodynamical moment $\vec{M}\left(M_{1}, M_{2}, M_{3}\right)$ are somewhat more complicated than the Lagally-force expressions given in the previous section and are given by (Landweber and Yih 1956).

$$
\begin{aligned}
& \left.M_{i}=\rho_{F} \forall\left[e_{i j k} \bar{x}_{j} \frac{d v_{k}}{d t}\right)-\frac{d}{d t^{*}} \sum_{j=1}^{3}\left(\Omega_{j} A_{3+i}, 3+j\right)\right]-4 \pi \rho_{F} \frac{d}{d t^{*}} \\
& \int_{S}\left\{\sigma_{3+i}\left[\sum_{j=i}^{3} x_{j} v_{j}-\phi_{s}\right]+\sigma_{s} \phi_{3+i}\right\} d S-4 \pi \rho_{F} e_{i j k} \int\left[\sigma x_{j}\left(v_{k}-v_{k}\right)\right] d S
\end{aligned}
$$

where $\left(\bar{x}_{1}, \bar{x}_{2}, \bar{x}_{3}\right)$ denote the position vector of the volume centroid of the body and $A_{3+i}, 3+j$ are the rotational added mass coefficients of the body. The surface source distribution here denoted by $\sigma_{3+i}$ and by $\sigma_{s}$ represent the image singularity system of the exterior velocity potentials $\phi_{3+i}$ and $\phi_{s}$ respectively. The sum of all surface source images corresponding to both the motion of the body and other boundaries and flow-producing mechanisms is represented by $\sigma$.

Since the ellipșoid has three mutual perpendicular planes of symmetry we have only three rotational added mass coefficients of the type $A_{3+i}, 3+i$, which corresponds to pure rotation about the $x_{i}$ axis, and in addition $\bar{x}_{i}=0$. Further- 
more, for the present case where the ellipsoid is maneuvering in an unbounded fluid medium without exterior flow-producing mechanisms, equation (37) may be simplified to yield the following expressions for the undisturbed Lagally moment $\vec{M}_{o}\left(M_{o 1}, M_{o 2}, M_{o 3}\right)$

$$
M_{o i}=-\rho_{F} \forall \frac{d}{d t^{*}}\left(\Omega_{i} A_{3+i}, 3+i\right)+4 \pi \rho_{F} e_{i j k} \int_{S} \sigma_{0} x_{j} v_{k} d S
$$

Substituting (29) and (33) in (38) and using (26) for the absolute time derivative yields the final expressions for $\vec{M}_{0}$,

$$
M_{o i}=-\rho_{F} F\left[A_{3+i}, 3+i \frac{d \Omega i}{d t}-B_{3+i} \Omega_{i+1} \Omega_{i+2}-B_{-3+i} V_{i+1} V_{i+2}\right]
$$

where we define for $i=1,2,3$.

$$
\begin{aligned}
& v_{3+i}=v_{i}, \Omega_{3+i}=\Omega_{i} ; A_{-3+i,-3+i}=A_{i i} ; A_{6+i}, 6+i=A_{3+i}, 3+i \\
& B_{i}=A_{i+1, i+1}-A_{i+2}, i+2
\end{aligned}
$$

Again the novelty in (39) is in the approach rather than in the result which was also given for example in Kochin et al. (1964,p. 401). Here we rederive the classlcal expression for the hydrodynamical moment acting on a maneuvering body with three orthogonal planes of symmetry in terms of its added masses, using the momentum approach which involves integration of the pressure over the surface rather than employing the energy (Kirchhoff's) method (Lamb 1932, p. 168). It should also be mentioned that the Lagally theorem is more general than the Kirchhoff-Lagrange method in the sense that it enables us to compute the forces and moments on Rankine bodies in cases where the latter method can not be 
applied. (Landweber and Yih 1956)

The three pure-rotational added mass coefficients $A_{3+i}, 3+i$ appear explicitly in the Lagally moment expression since there exists no generalization of the Taylor added mass theorem for pure rotation which relates the added masses to the corresponding image singularity system. However, by applying the kineticenergy theorem (Lamb 1932, p. 153-155) one can express the rotational added masses of an ellipsoid in terms of the coefficients $c_{2}^{i}$ defined in (18) (Miloh 1973) as

$$
A_{3+i, 3+i}=-\frac{\left(a_{i+1}^{2}-a_{i+2}^{2}\right)^{2}}{5\left(a_{i+1}^{2}+a_{i+2}^{2}\right)}\left[1+\frac{20 \pi c_{2}^{6-i}}{3 ¥\left(a_{i+1}^{2}+a_{i+2}^{2}\right)}\right]
$$

The coefficients $C_{2}^{i}$ in turn may also be expressed in terms of the tabulated incomplete Legendre elliptic functions (Miloh 1973).

\section{HYDRODYNAMICAL FORCES IN DISTURBED OR BOUNDED FLUID DOMAIN}

In this section we derive expressions for the Lagally forces acting on a maneuvering ellipsoid in the presence of exterior disturbance resulting from the proximity of other bodies or boundaries as well as isolated flow-producing singularities. The external boundaries will be represented by a surface distribution of sources which may be continuous or discrete. We will consider only sources as a model for treating exterior flow-producing singularities where it is understood that higher order singularities may also be handled by the same method by a proper differentiation. It was found instructive to derive the first expression for the Lagally forces resulting from the presence of an exterior source, before dealing with the more general problem concerning arbitrary fluid disturbances. 
Consider an isolated source of strength $\delta(t)$ lying at $M^{\prime}\left(\rho^{\prime}, \mu^{\prime}, U^{\prime}\right)$ such that $\rho^{\prime}>a_{1}$, which implies that the source lies in the domain exterior to the canonical ellipsoid. The source may translate in an arbitrary manner such that the instantaneous location of the source in a Cartesian coordinate system attached to the ellipsoid is $M^{\prime}\left(\vec{r}^{\prime}\right)$ where $\vec{r}^{\prime}\left(x_{1}^{\prime}, x_{2}^{\prime}, x_{3}^{\prime}\right)$ is a time dependent radius vector.

It is convenient to decompose the Lagally force $\vec{T}\left(T_{1}, T_{2}, T_{3}\right)$ acting on a maneuvering ellipsoid in the presence of an exterior disturbance into three terms

$$
\mathrm{T}_{i}=\mathrm{T}_{\mathrm{oi}}+\mathrm{T}_{\mathrm{pi}}+\mathrm{T}_{\mathrm{qi}}
$$

where as before $\overrightarrow{\mathrm{T}}_{0}\left(\mathrm{~T}_{\circ 1}, \mathrm{~T}_{\circ 2}, \mathrm{~T}_{\circ 3}\right)$ denotes the force acting on the ellipsoid when the exterior disturbance is not present, $\vec{T}_{\mathrm{p}}\left(\mathrm{T}_{\mathrm{p} 1}, \mathrm{~T}_{\mathrm{p} 2}, \mathrm{~T}_{\mathrm{p} 3}\right)$ is the force acting on a stationary ellipsoid where the exterior disturbances is moving and $\vec{T}_{q}\left(T_{q 1}, T_{q 2}, T_{q 3}\right)$ is the contribution from the interaction between the motions of both the ellipsoid and the exterior disturbances.

Applying the general Lagally formula (25) for the case where the exterior disturbance is an isolated source, yields the following expressions for $\overrightarrow{\mathrm{T}}_{\mathrm{p}}$ and $\overrightarrow{\mathrm{T}}_{\mathrm{q}}:$

$$
T_{p i}=4 \pi \rho_{F} \frac{d}{d t} \int_{S} \sigma_{s} x_{i} d S-4 \pi \rho_{F} \int_{S} \sigma_{s} v_{i} d S
$$

and

$$
T_{q i}=4 \pi \rho_{F} e_{i j k} \Omega_{j} \int \sigma_{s} x_{k} d S-4 \pi \rho_{F} \int \sigma_{o} v_{i} d S
$$


where $\sigma_{S}\left(M_{e}, M^{\prime}\right)$ is the image source distribution induced at $M_{e}$ due to the exterior source lying at $M^{\prime}$ and is given by (20) multiplied by the source strength $\delta$. The image distribution for maneuvering ellipsoid in unbounded medium $\sigma_{0}\left(\mathrm{M}_{\mathrm{e}}\right)$ appearing in (45) is given by (24). The velocity induced at $M_{e}$ due to the presence of the source at $M^{i}$ is denoted by $\vec{v}\left(v_{1}, v_{2}, v_{3}\right)$ and may be expressed as,

$$
v_{i}\left(M_{e}, M^{\prime}\right)=-\left.\delta \frac{\partial}{\partial x_{i}}\left[\frac{1}{R\left(M, M^{\prime}\right)}\right]\right|_{M_{M} M_{e}}=\delta \frac{\partial}{\partial x_{i}^{\prime}}\left[\frac{1}{R\left(M_{e}, M^{\prime}\right)}\right]
$$

or, after substituting (16) into (46)

$$
v_{i}\left(M_{e}, M^{\prime}\right)=4 \pi \delta \frac{\partial}{\partial x_{i}^{\prime}} \sum_{n=0}^{\infty} \sum_{m=1}^{2 n+1} \frac{P_{n}^{m}\left(M_{e}\right)}{(2 n+1) \gamma_{n}^{m}} Q_{n}^{m}\left(M^{\prime}\right)
$$

Let us turn now to the computation of the various integrals occuring in (44) and (45). Substitution of (28) and (42) into the first integrals in the right had side of (44) yields after applying the orthogonality properties of the Lame functions

$$
\int_{S} \sigma_{s} x_{i} d s=-\delta \alpha_{i}\left(M^{\prime}\right)
$$

Similarly, substitution of (28) and (47) renders for the second integral in (44) 


$$
\int \sigma_{s} v_{i} d S=-2 \pi \delta^{2} \frac{\partial}{\partial x_{i}^{\prime}} \sum_{n=0}^{2 n+1} \sum_{m=1}^{m} D_{n}^{m}\left[Q_{n}^{m}\left(M^{\prime}\right)\right]^{2}
$$

$\mathrm{S}$

lere $D_{n}^{m}$ has been defined in (18).

The above relations yield the final form for the disturbance Lagally

orce $\overrightarrow{\mathrm{T}}_{\mathrm{p}}$,

$$
T_{p i}=4 \pi \rho_{F} \frac{d}{d t}\left[\delta \alpha_{i}\left(M^{\prime}\right)\right]-8 \pi^{2} \rho_{F} \delta^{2} \frac{\partial}{\partial x_{i}^{\prime}} \sum_{n=0}^{\infty} \sum_{m=1}^{2 n+1} D_{n}^{m}\left[Q_{n}^{m}\left(M^{\prime}\right)\right]^{2}
$$

The interaction force $\overrightarrow{\mathrm{T}}_{\mathrm{q}}$ will be consldered next. Substituting (24) nd (47) into (45) yields

$$
\int \sigma_{0} v_{i} d S=-\delta \frac{\partial}{\partial x_{1}^{\prime}} \sum_{i=1}^{3}\left[v_{1} \alpha_{i}\left(M^{\prime}\right)+\Omega_{i} \beta_{i}\left(M^{\prime}\right)\right]
$$

$\mathrm{S}$

inally, applying (48) we get the following expression for the interaction orce

$$
T_{q i}=4 \pi \rho_{F} \delta e_{1 j k} \Omega_{j} \alpha_{k}\left(M^{\prime}\right)+4 \pi \rho_{F} \delta \frac{\partial}{\partial x_{i}^{\prime}} \sum_{i=1}^{3}\left[v_{1} \alpha_{1}\left(M^{\prime}\right)+\Omega_{1} \beta_{i}\left(M^{\prime}\right)\right]
$$

'he general expression for the Lagally force acting on a maneuvering ellipsoid in the preence of exterior source where both its strength and location are time depenlent, is found by summing the different contributions given by (34) (50) and 52) namely 


$$
T_{i} / 4 \pi \rho_{F}=\frac{d}{d t}\left[\delta \alpha_{i}\left(M^{\prime}\right)-\forall A_{i i} v_{i}\right]+e_{i j k} \Omega_{j} \quad\left[\delta \alpha_{k} \quad\left(M^{\prime}\right)-\forall A_{k k} v_{k}\right]
$$

$$
+\frac{\partial}{\partial x_{1}^{\prime}}\left\{\sum_{j=1}^{3}\left[v_{j} \alpha_{j}\left(M^{\prime}\right)+\Omega_{j} \beta_{j}\left(M^{\prime}\right)\right]-2 \pi \delta \sum_{n=0}^{\infty} \sum_{m=1}^{2 n+1} D_{n}^{\mathfrak{m}}\left[Q_{n}^{m}\left(M^{\prime}\right)\right]^{2}\right\}
$$

Once the expression for the Lagally force acting on a maneuvering ellipsold by an exterior source has been determined it is easy to calculate the corresponding force due to an arbitrary exterior disturbance. Let us assume that the exterior disturbance is represented by a continuous or discrete source distribution $\delta^{\prime}\left(M^{\prime}, t\right)$ over the surface $S^{\prime}$ which may be finite or infinite such that the velocity potential induced at points on the canonical ellipsoid is given by

$$
\Phi\left(M_{e}\right)=-\int_{S^{\prime}} \delta^{\prime}\left(M^{\prime}, t\right) \frac{d S^{\prime}}{R\left(M^{\prime}, M_{e}^{\prime}\right.}
$$

Here $S^{\prime}$ is the set of all points $M^{\prime}$. For the case of discrete singularities the integral in (54) may be replaced by a summation over ail singularities. For the present case equation (53) may be generalized to yield

$T_{i} / 4 \pi \rho_{F}=\frac{d}{d t}\left[\int_{S^{\prime}} \delta^{\prime} \alpha_{i}\left(M^{\prime}\right) d S^{\prime}-\forall A_{i 1} V_{1}\right]+e_{1 j k_{j}}\left[\int_{S^{\prime}} \delta^{\prime} \alpha_{k}\left(M^{\prime}\right) d S^{\prime}-\nabla A_{k k} V_{k}\right]$

$$
\begin{aligned}
& +\int_{S^{\prime}} \delta^{\prime} \sum_{j=1}^{3} \frac{\partial}{\partial x_{i}^{\prime}}\left[v_{j} \alpha_{j}\left(M^{\prime}\right)+\Omega_{j} \beta_{j}\left(M^{\prime}\right)\right] d S^{\prime} \\
& -2 \pi \int_{S^{\prime}} \int_{S^{\prime \prime}} \delta^{\prime} \delta^{\prime \prime} \sum_{n=0}^{\infty} \sum_{m=1}^{2 n+1} D_{n}^{m} Q_{n}^{m}\left(M^{\prime \prime}\right) \frac{\partial}{\partial x_{1}^{\prime}}\left[Q_{n}^{m}\left(M^{\prime}\right)\right] d S^{\prime} d S^{\prime \prime}
\end{aligned}
$$


where $S^{\prime \prime}=S^{\prime}$ and $M^{\prime \prime}=M^{\prime}$.

On the assumption that the singularities representation of the exterior disturbance $\delta^{\prime}$ is known the above expression enables us to calculate directly the force on a maneuvering ellipsoid in the proximity of an arbitrary disturbance. The first term in (55) represents the effect of the unsteadiness of the problem, the next two terms the additional force caused by the motion of the ellipsoid. The last term is the interaction force which is also the force on a stationary ellipsoid due to stationary external disturbance. It arises from the fact that the singularities of the external disturbance have an image within the ellipsoid. The interaction force is usually small in comparison with the terms arising from the motion of the ellipsoid.

\section{HYDRODYNAMICAL MOMENT IN DISTURBED OR BOUNDED FLUID DOMAIN}

In a similar manner we start with the derivation of the Lagally moment acting on a maneuvering ellipsold in the presence of an exterior source. Again we decompose the Lagally moment $\vec{M}\left(M_{1}, M_{2}, M_{3}\right)$ into three terms.

$$
M_{1}=M_{o i}+M_{p i}+M_{q 1} \quad 1=1,2,3
$$

where $\vec{M}_{0}\left(M_{o 1}, M_{o 2}, M_{o 3}\right)$ denotes the moment in undisturbed medium, $\vec{M}_{p}\left(M_{p 1}, M_{p 2}, M_{p 3}\right)$ is the moment acting on a stationary ellipsold and $\overrightarrow{\mathrm{M}}_{\mathrm{q}}\left(\mathrm{M}_{\mathrm{q} 1}, \mathrm{M}_{\mathrm{q} 2}, \mathrm{M}_{\mathrm{q} 3}\right)$ is the additional moment caused by the motion of the ellipsoid in the proximity of the disturbance.

The undisturbed Lagally moment $\overrightarrow{\mathrm{M}}_{0}$ has been derived in (39) and for $\vec{M}_{p}$ and $\vec{M}_{q}$ we may write the following (37), 


$$
M_{p i}=-4 \pi \rho_{F} e_{i j k} \int_{S} \sigma_{s} x_{j} V_{k} d S
$$

and

$$
M_{q i}=-4 \pi \rho_{\dot{F}} \cdot \frac{d}{d t *} \int_{S}\left(\sigma_{s} \phi_{3+i}-\phi_{3+1} s\right) d S-4 \pi \rho_{F} e_{1 j k} \int_{s} x_{j}\left(\sigma_{0} v_{k}-\sigma_{s} v_{k}\right) d S
$$

The value of $\vec{M}_{p}$ will be considered first. The following relation may be easily verified.

$$
e_{i j k} x_{j} \frac{\partial}{\partial x_{k}}\left[\frac{1}{R\left(M, M^{\prime}\right)}\right]=e_{1 j k} x_{j}^{\prime} \frac{\partial}{\partial x_{k}}\left[\frac{1}{R\left(M, M^{\prime}\right)}\right]
$$

Where here $M\left(x_{1}, x_{2}, x_{3}\right)$ and $M^{\prime}\left(x_{1}^{\prime}, x_{2}^{\prime}, x_{3}^{\prime}\right)$. Substituting $(46),(47)$ and (59) into (57) one gets

$$
M_{p i}=-4 \pi \rho_{F} e_{i j k} x_{j}^{\prime} \int_{S} \sigma_{s} v_{k} d S
$$

Which in view of (49) yields

$$
M_{p i}=8 \pi^{2} \rho_{F} \delta^{2} e_{i j k} x_{j}^{\prime} \frac{\partial}{\partial x_{k}^{\prime}} \sum_{n=0}^{\infty} \sum_{m=1}^{2 n+1} D_{n}^{m}\left[Q_{n}^{m}\left(M^{\prime}\right)\right]^{2}
$$

The interaction moment $\vec{M}_{q}$ is considered next. From (21) and (24) we have the following expressions;

$$
\phi_{3+i}\left(M_{e}\right)=\beta_{i}\left(M_{e}\right)
$$


Substituting (64) and (66) into (58) yields the following expression for the interaction moment.

$M_{q 1} / 4 \pi \rho_{F}=\frac{d}{d t}\left[\delta \beta_{i}\left(M^{\prime}\right)\right]+\delta e_{i j k}\left\{v_{k} \alpha_{j}\left(M^{\prime}\right)+\Omega_{j} \beta_{k}\left(M^{\prime}\right)+x_{j}^{\prime} \frac{\partial}{\partial x_{k}^{\prime}} \sum_{j=1}^{3}\left[v_{j} \alpha_{j}\left(M^{\prime}\right)+\Omega_{j} \beta_{j}\left(M^{\prime}\right)\right]\right\}$

The final expression for the Lagally moment acting on a maneuvering ellipsoid in the proximity of an exterior source is obtained by summing up the three different contributions to the moment given in (39), (61) and (67) which yields

$$
\begin{aligned}
& M_{i} / 4 \pi \rho_{F}=\frac{d}{d t}\left[-\frac{\forall}{4 \pi} A_{3+i, 3+i} \Omega_{i}+\delta B_{i}\left(M^{\prime}\right)\right]+\frac{\forall}{4 \pi}\left[B_{3+1} \Omega_{i+1} \Omega_{i+2}+B_{-3+1} V_{i+i} V_{i+2}\right] \\
& +\delta e_{i j k}\left[v_{k} \alpha_{j}\left(M^{\prime}\right)+\Omega_{j} \beta_{k}\left(M^{\prime}\right)\right]+\delta e_{i j k} x_{j}^{\prime} \frac{\partial}{\partial x_{k}^{\prime}}\left\{\sum_{j=1}^{3}\left[V_{j} \alpha_{j}\left(M^{\prime}\right)+\Omega_{j} \beta_{j}\left(M^{\prime}\right)\right]\right. \\
& \left.+2 \pi \delta \sum_{n=0}^{\infty}{ }_{m=1}^{2 n+1} D_{n}^{m}\left[Q_{n}^{m}\left(M^{\prime}\right)\right]^{2}\right\}
\end{aligned}
$$

For the general case where the velocity potential induced by the exterior disturbance is given by (54) the expression for the Lagally moment becomes

$M_{i} / 4 \pi \rho_{F}=\frac{d}{d t}\left[-\frac{\forall}{4 \pi} A_{3+i, 3+i} \Omega_{1}+\int_{S^{\prime}} \delta^{\prime} \beta_{i}\left(M^{\prime}\right) d S^{\prime}\right]+\frac{\forall}{4 \pi}\left[B_{3+i} \Omega_{1+1} \Omega_{1+2}+\right.$ 
and

$$
\sigma_{3+i}\left(M_{e}\right)=-\frac{5 a_{i} \beta_{i}\left(M_{e}\right)}{3 \forall F_{2}^{6-i}\left(a_{1}\right) \sqrt{\left(a_{1}^{2}-\mu^{2}\right)\left(a_{1}^{2}-v^{2}\right)}}
$$

The disturbance velocity potential $\phi_{s}\left(M_{e}, M^{\prime}\right)$ and its 1mage system of singularities induced at the surface of the ellipsoid $\sigma_{s}\left(M_{e}, M^{\prime}\right)$ are given by (17) and (20) respectively after multiplication by the source strength $\delta$. Substituting (17), (20), (62) and (64) into the first integral in (58) yie1ds

$$
\int_{S}\left(\sigma_{s} \phi_{3+i}-\sigma_{3+i} \phi_{s}\right) d s=-\delta B_{1}\left(M^{\prime}\right)
$$

The second integral on the right hand side of (59) may be also written as

$$
e_{i j k} \int_{S} x_{j}\left(\sigma_{0} v_{k}-\sigma_{s} v_{k}\right) d S=e_{i j k}\left[x_{j}^{\prime} \int_{S} \sigma_{o} v_{k} d S-v_{k} \int_{S} \sigma_{s} x_{j} d S\right]
$$

In the above form the relations given in (48) and (51) are readily used to give

$$
e_{i j k} \int_{S} x_{j}\left(\sigma_{o} v_{k}-\sigma_{s} v_{k}\right) d S=-\delta e_{i j k}\left\{v_{k} \alpha_{j}\left(M^{\prime}\right)+x_{j}^{\prime} \frac{\partial}{\partial x_{k}^{\prime}} \sum_{j=1}^{3}\left[v_{j} \alpha_{j}\left(M^{\prime}\right)+\Omega_{j} \beta_{j}\left(M^{\prime}\right)\right]\right\}
$$




$$
\begin{aligned}
& \left.+B_{-3+i} v_{i+i} v_{i+2}\right]+e_{i j k} \int_{S^{\prime}} \delta^{\prime}\left[v_{k} \alpha_{j}\left(M^{\prime}\right)+\Omega_{j} \beta_{k}\left(M^{\prime}\right)\right] d S^{\prime} \\
& +e_{i j k} x_{j}^{1} \int_{S^{\prime}} \delta^{\prime} \sum_{j=1}^{3} \frac{\partial}{\partial x_{k}^{\prime}}\left[v_{j} \alpha_{j}\left(M^{\prime}\right)+\Omega_{j} \beta_{j}\left(M^{\prime}\right)\right] d^{\prime} \\
& +2 \pi e_{i j k} x_{j}^{\prime} \int_{S^{\prime}} \int_{S^{\prime \prime}} \delta^{\prime} \delta^{\prime \prime} \sum_{n=0}^{\infty} \sum_{m=1}^{2 n+1} D_{n}^{m} Q_{n}^{m}\left(M^{\prime \prime}\right) \frac{\partial}{\partial x_{k}^{\prime}}\left[Q_{n}^{m}\left(M^{\prime}\right)\right] d S^{\prime} d^{\prime \prime}
\end{aligned}
$$

Thus the hydrodunamical moment acting on a maneuvering ellipsoid in a bounded or disturbed medium may be calculated directly from (69) once the source singularities representation of the exterior disturbance is specified. As before, for discrete source distribution the integral signs in (69) are replaced by a summation over all singularities. The derived expressions for the Lagally force and moment may be also modifled to account for higher order singularities by a proper partial differentiation of the terms under the integral sign.

\section{MODEL EXAMPLE: PROPELLER-HULL INTERACTION}

As a demonstration of the general expressions thus given for the hydrodynamical forces and moments experienced by a maneuvering tri-axial ellipsoid, we consider the effect of placing a thrust-producing mechanism on the major axis of a self-propelled ellipsoid. In addition, we exclude rotational motion of the ellipsoid and consider only translation in the direction of the major axis. The aim is to determine the effect of such a thrust-producing system on the flow pattern about the hull, with special reference to the determination of basic parameters such as wake fraction, thrust deduction and propeller-hull exciting forces. 
Following the classical work of Dickmarn (1938) the hydrodynamical effect of a single propeller located at the ship's stern may be represented by a point sink in accordance with the actuator disk theory. The strength of the sink, lying at $x=x_{p}>a_{1} ; y=z=0$ (or $\rho=x_{p}>a_{1} ; \mu=k ; \nu=h_{,}$) is denoted by $\delta(t)<0$. The time dependent output of the sink may be also written as

$$
\delta(t)=\bar{\delta}+\tilde{\delta} e^{i b \omega t}
$$

where $\bar{\delta}$ denotes the mean (steady) output of the sink and $\tilde{\delta}$ is the amplitude of the harmonic time dependent variation of the sink output. The angular velocity of the propeller is denoted by $\omega$ and $b$ is the number of blades.

The mean sink strength may also be expressed in terms of the thrust loading coefficient $\overline{\mathrm{C}}_{\mathrm{TH}}$, thus following Dickmann's analysis,

$$
\bar{\delta}=-\frac{A \bar{V}}{4 \pi}\left[\left(1+\bar{C}_{\mathrm{TH}}\right)^{1 / 2}-1\right]
$$

where $A$ is the propeller area taken as a disk excluding the hub, and the thrust loading coefficient is defined by

$$
\overline{\mathrm{C}}_{\mathrm{TH}}=\frac{2 \overline{\mathrm{T}}_{\mathrm{h}}}{\rho \overline{\mathrm{V}}^{2} \mathrm{~A}}
$$

Here $\bar{T}_{h}$ denotes the total mean thrust required to attain a constant cruising velocity $\bar{V}$. For a propeller operating in the wake behind a hull, a better model for the sink strength is believed to be the one suggested by Nowacki and Sharma (1971) in which equation (71) is augmented by 


$$
\bar{\delta}=-\frac{A \bar{V}}{4 \pi}\left(1-\overline{\mathrm{W}}_{\mathrm{e}}\right)\left[\left(1+\overline{\mathrm{C}}_{\mathrm{TH}}\right)^{1 / 2}-1\right]
$$

The mean effective wake fraction $\overline{\mathrm{W}}_{e}$ is defined as the ratio between the mean decremental velocity over the propeller disk in the presence of the propeller and the advance velocity. The presence of a propeller behind the hull will also alter the pressure field about the hull giving rise to an additional interaction force between the propeller and the hull. Because of that reason, the propeller must develop a larger thrust in the case of a self-propelled hull than the thrust needed when the hull is towed. The ratio between the propellerhull interaction force and the total propeller thrust is the so called thrust deduction coefficient $\lambda_{\mathrm{p}}$.

$$
\lambda_{p}=\frac{T_{1}}{T_{h}}
$$

where $\mathrm{T}_{1}$ denotes the absolute value of the propeller induced deduction-thrust on the hull. In the present demonstration we wish to examine the relation between the thrust deduction … and the effective wake fraction for ellipsoidal hulls.

Since both the hull and the propeller move with identical velocity $V(t)$ in the direction of the negative $x_{1}$ direction, the interaction force between the propeller and the hull is given by (53),

$$
\begin{gathered}
\frac{T_{1}}{4 \pi \rho_{F}}=-\alpha_{1}\left(M^{\prime}\right) \frac{d \delta}{d t}+\frac{y}{4 \pi} A_{11} \frac{d V}{d t}-\delta V \frac{d a_{1}\left(M^{\prime}\right)}{d x_{1}}+2 \pi \delta^{2} \sum_{n=0}^{\infty} \sum_{m=1}^{2 n+1} D_{n}^{m} \frac{d}{d x_{1}}\left[Q_{n}^{m}\left(M^{\prime}\right)\right]^{2} \\
x_{1}=x_{p}
\end{gathered}
$$




$$
\frac{\bar{T}_{1}}{4 \pi \rho_{F}}=-\delta V \frac{d \alpha_{1}\left(M^{\prime}\right)}{d x_{1}}+2 \pi \delta^{2} \sum_{n=0}^{\infty} \sum_{m=1}^{2 n+1} D_{n}^{m} \frac{d}{d x_{1}}\left[Q_{n}^{m}\left(M^{\prime}\right)\right]^{2} \mid x_{1}=x_{p}
$$

which is also the steady component of the thrust deduction. For most practical purposes $\mathrm{V} \gg \bar{\delta}$ and it is sufficient to consider only the first non-vanishing term in the double summation in (76) (assuming that the series converges uniform1y). For $n=0$ there is no contribution from the double series since $\mathrm{D}_{\mathrm{O}}^{1}=0$ and the only non-vanishing term for $\mathrm{n}=1$ corresponds to $\mathrm{m}=1$. Thus equation (76) together with (18), (22) and (30) yields

$$
\frac{\bar{T}_{1}}{4 \pi \rho_{F}} \simeq-\left.\frac{\delta C_{1}^{1}}{h k} \cdot \frac{d}{d x_{1}} Q_{1}^{1}\left(M^{\prime}\right)\left[V-\frac{\delta Q_{1}^{1}\left(M^{\prime}\right)}{h k}\right]\right|_{x_{1}}=x_{p}
$$

where (Miloh 1974)

$$
Q_{1}^{1}\left(M^{\prime}\right)=3 h k x_{p} \int_{x_{p}}^{\infty} \frac{d \rho}{\rho^{2} \sqrt{\left.\left(\rho^{2}-h^{2}\right) \rho^{2}-k^{2}\right)}}=\frac{3 x_{p}}{k}[F(\theta, \lambda)-E(\theta, \lambda)]
$$

Here $F$ and $E$ denote the Legendre incomplete elliptic integrals of the first and second kind respectively (Byrd and Friedman 1971) with arguments given by,

$$
\theta=\sin ^{-1} \frac{k}{x_{p}} ; \lambda=\frac{h}{k}
$$




$$
\left.\frac{\mathrm{d}}{\mathrm{dx} \mathrm{x}_{1}} \mathrm{Q}_{1}^{1}\left(\mathrm{M}^{\prime}\right)\right|_{\mathrm{x}_{1}=\mathrm{x}_{\mathrm{p}}}=\frac{3}{\mathrm{k}}[\mathrm{F}(\theta, \lambda)-\mathrm{E}(\theta, \lambda)]-\frac{3 h \mathrm{k}}{\mathrm{x}_{\mathbf{p}} \sqrt{\left(\mathrm{x}_{\mathrm{p}}^{2}-\mathrm{h}^{2}\right)\left(\mathrm{x}_{\mathrm{p}}^{2}-\mathrm{k}^{2}\right)}}
$$

The additional coefficient in (77) namely $c_{1}^{1}$ is defined in (33) in terms of the logitudinal added mass coefficient of the ellipsold, $A_{11}$ and may be also expressed, in a similar manner to (80) by,

$$
\frac{1}{C_{1}^{1}}=\frac{3}{h_{k}^{2}}[F(\theta * \lambda)-E(\theta \star \lambda)]-\frac{3}{a_{1} a_{2} a_{3}}
$$

where here $\theta *=\sin ^{-1} \frac{k}{a}$. It should be noted that the r.h.s. of (81) equals the r.h.s. of (80) divided by hk where $x_{p}$ is replaced by $a_{1}$. Thus, equation (77) is an anlytic expression for the thrust deduction in terms of the propeller output and its location along the major axis and the hull geometry. The various functions in (77) are given in (78), (80) and (81) in terms of the tabulated Legendre elliptic integrals which are suitable forms for numerical computation.

In order to determine the magnitude of the effective wake fraction, we apply equations (17) and (23) which render

$$
\bar{W}_{e}=\frac{C_{1}^{1}}{h k} \frac{d_{1}^{1}\left(M^{\prime}\right)}{d x_{1}}-2 \pi(\bar{\delta} / V) \sum_{n=0}^{\infty} \sum_{m=1}^{2 n+1} D_{n}^{m} \frac{d}{d x_{1}}\left[\left.\left.Q_{n}^{m}\left(M^{\prime}\right)\right|^{2}\right|_{x_{1}=x_{p}}\right.
$$

Comparing (76) and (82) implies that the relation between the thrust deduction and the effective wake fraction is given by,

$$
\overline{\mathrm{T}}_{1}=-4 \pi \rho_{\mathrm{F}} \overline{\delta \mathrm{V}} \overline{\mathrm{W}}_{\mathrm{e}}
$$


which may also be obtained directly by considering the Lagally force on the sink in the presence of the wake.

The non-dimensional representation of (83) is

$$
\lambda_{p}=\frac{2 \bar{W}_{e}\left(1-\bar{W}_{e}\right)}{1+\sqrt{1+C_{T H}}}
$$

which is obtained by substituting (72), (73) and (74) in (83). The above relation between the effective wake fraction, the thrust deduction coefficient and thrust loading coefficient is exact within the limits of potential flow theory and Dickmann's model of the propeller. A similar relation, aside from the correction term $1-\bar{W}_{e}$, has been given by Tsakonas and Jacobs (1960) in their study of prolate spherold. Tsakonas and Jacobs derivation ignores the contribution of images in the body which are required to maintain its outline in the presence of the propeller singularity. It should be noted that we were able to show that the relation holds also in the case where the propeller images are taken into account, thus the relation between the thrust-deduction and the wake fraction has new meaning. It is true that for elongated slender bodies Kroukovsky (1956) was able to show that the effect of the propeller images is small, but on the other hand Martinek and Yeh (1956) pointed out that this effect is Important and may in fact explain some of the discrepancies between theory and experiments especially for blunt bodies.

Equation (82) can be solved explicitly for the effective wake fraction when using the definition (73) for the sink strength, yielding, 


$$
\bar{W}_{e}=\frac{\left[\frac{C_{1}^{1}}{h k} \frac{d}{d x_{1}} Q_{1}^{1}\left(M^{\prime}\right)+4 \pi h k A^{*} \sum_{n=0}^{\infty} \sum_{m=1}^{2 n+1} D_{n}^{m} Q_{n}^{m}\left(M^{\prime}\right) \frac{d}{d x_{1}} Q_{n}^{m}\left(M^{\prime}\right)\right]}{\left[1+4 \pi h k A \sum_{n=0}^{\infty} \sum_{m=1}^{2 n+1} D_{n}^{m} Q_{n}^{m}\left(M^{\prime}\right) \frac{d}{d x_{1}} Q_{n}^{m}\left(M^{\prime}\right)\right]}
$$

where $A^{*}=\frac{A}{4 \pi h k}\left[\sqrt{1+C_{T H}}-1\right]$ is a non-dimensional parameter which is small compared to unity for lightly loaded propellers and for elongated hulls. Numerical experience with the double series in (85) shows that for $\rho^{\prime}>a_{1}$ the series converges fast and it is sufficient to consider only the first term in the series which corresponds to $n=1$ and $m=1$. For $n=2$ there are only two non-zero coefficients corresponding to $m=1$ and $m=2$. Hence equation (85) can be approximated by

$$
\overline{\mathrm{W}}_{\mathrm{e}}=\frac{\mathrm{C}_{1}^{1}}{\mathrm{hk}} \frac{\mathrm{d}}{\mathrm{dx}} \mathrm{Q}_{1}^{1}\left(\mathrm{M}^{\prime}\right) \quad\left\{1+\mathrm{A}^{*} Q_{1}^{1}\left(M^{\prime}\right)\left[1-\frac{\mathrm{C}_{1}^{1}}{\mathrm{hk}} \frac{\mathrm{d}}{\mathrm{dx}} \mathrm{Q}_{1}^{1}\left(\mathrm{M}^{\prime}\right)\right]\right\}
$$

where the coefficient $c_{1}^{1}$, the ellipsoidal harmonic $Q_{1}^{1}\left(M^{\prime}\right)$ and its partial derivative with respect to $x_{1}$ evaluated at $x_{1}=x_{p}$, are given by (78), (79) and (81).

For prolate spheroidal shapes, $a_{2}=a_{3}$ or $h=k$ and equation (86) reduces to,

$$
=\frac{\log \frac{x_{p}-h}{x_{p}+h}+\frac{2 h x_{p}}{x_{p}^{2}-h^{2}}}{\log \frac{a_{1}-h}{a_{1}+h}+\frac{2 h a_{1}}{a_{1}^{2}-h^{2}}}\left\{1-\frac{3}{2} A^{*}\left[\frac{x_{p}}{h} \log \frac{x_{p}-h}{x_{p}+h}+2\right]\left[1-\frac{\log \frac{x_{p}-h}{x_{p}+h}+\frac{2 h x_{p}}{x_{p}^{2}-h^{2}}}{\log \frac{a_{1}-h}{a_{1}+h}+\frac{2 h a_{1}}{a_{1}^{2}-h^{2}}}\right\}\right.
$$


When $\rho^{\prime} \rightarrow a_{1}$ the term multiplying the parenthesis of (86) is unity implying that $\vec{W}_{e}\left(\rho^{\prime} \rightarrow a_{1}\right)=1$. Similarly for large values of $\rho^{\prime}$ we get $\overline{\mathrm{W}}_{\mathrm{e}}\left(\rho^{\prime} \rightarrow \infty\right)=0$. Hence the thrust decudtion coefficient as given in (84), vanishes in both limits as $\rho^{\prime \rightarrow a_{1}}$ and $\rho^{\prime \rightarrow \infty}$ and has a local maximum of $\frac{1}{2}\left[1+\sqrt{1+\bar{C}_{\mathrm{TH}}}\right]^{-1} \quad\left(0.25\right.$ for lightly loaded propellers) at $\overline{\mathrm{W}}_{\mathrm{e}}=0.5$.

The time dependent component of the thrust deduction which induces the hull vibrations is found by substituting (70) in (75) and considering timeharmonic terms of blade frequency $b \omega$,

$$
\begin{aligned}
\frac{\tilde{T}_{1}}{4 \pi \rho_{F}}=-\alpha_{1}\left(M^{\prime}\right) \frac{d}{d t}\left(\tilde{\delta} e^{i b \omega t}\right)-\tilde{\delta} V e^{i b \omega t} \frac{d \alpha_{1}\left(M^{\prime}\right)}{d x_{1}} \\
+4 \pi \delta \tilde{\delta} e^{i b \omega t} \sum_{n=0}^{\infty} \sum_{m=1}^{2 n+1} D_{n}^{m} \frac{d}{d x_{1}}\left[Q_{n}^{m}\left(M^{\prime}\right)\right]^{2}
\end{aligned}
$$

where only the real part has to be considered.

In deriving (88) it was also assumed that the ship longitudinal acceleration $\frac{\mathrm{dV}}{\mathrm{dt}}$ is negligible due to the large inertia of the ship. The resulting relations for the vibratory force, when considering only the first nonvanishing term in the infinite series of (88), may be expressed as,

$$
\tilde{T}_{1}=4 \pi \rho_{F} \theta e^{i(b \omega t+\theta)}
$$

where $\theta$ and $\theta$ are the amplitude and the phase angle of the vibratory force respectively, which are given by

$$
\theta=-\tilde{\delta} \frac{c_{1}^{1}}{h k} \sqrt{\theta_{1}^{2}+\theta_{2}^{2}} \quad, \quad \theta=\mathrm{tg}^{-1}\left(\theta_{2} / \theta_{1}\right)
$$


and

$$
\begin{aligned}
& \Theta_{1} \equiv \frac{d}{d x_{1}} Q_{1}^{1}\left(M^{\prime}\right)\left[V-2 \delta Q_{1}^{1}\left(M^{\prime}\right)\right] \\
& \theta_{2}=b \omega Q_{1}^{1}\left(M^{\prime}\right)
\end{aligned}
$$

For a spheroidal shape $(k+h)$ equation $(90)$ reduces to

$$
\theta=\frac{2 \tilde{\delta}}{3\left[\log \frac{a_{1}-h}{a_{1}+h}+\frac{2 h a_{1}}{a_{1}^{2}-h^{2}}\right]} \sqrt{\theta_{1}^{2}+\theta_{2}^{2}}
$$

where,

$$
\begin{aligned}
& \Theta_{1}=\frac{3}{2 h}\left[\log \frac{x_{p}-h}{x_{p}+h}+\frac{2 h x_{p}}{x_{p}^{2}-h^{2}}\right]\left\{v+3 \delta\left[\frac{x_{p}}{h} \log \frac{x_{p}-h}{x_{p}+h}+2\right]\right\} \\
& \Theta_{2}=-\frac{3}{2} b \omega\left[\frac{x_{p}}{h} \log \frac{x_{p}-h}{x_{p}+h}+2\right]
\end{aligned}
$$

The limiting value of (93) as $h \rightarrow 0$ is also of practical interest since it represents a spherical shape of radius $a_{1}$. By using L'Hospital's rule it is possible to remove the indeterminacies occurring in (93), (94) and (95) in the limit $h \rightarrow 0$ which result in the following expressions for propeller induced vibratory force given in (89).

$$
\tilde{\mathrm{T}}_{1}=\pi \tilde{\delta} \rho_{F}\left(\frac{\mathrm{a}_{1}}{\mathrm{x}_{\mathrm{p}}}\right)^{3}\left(4 \mathrm{~V}^{2}+\mathrm{b}^{2} \omega^{2} \mathrm{x}_{\mathrm{p}}^{2}\right)^{\frac{1}{2}} \mathrm{e}^{1(b \omega t+\theta)}
$$


where the phase angle $\theta$ is given by

$$
\theta=\operatorname{tg}^{-1}\left(-\frac{b \omega x_{p}}{2 V}\right)
$$

In a similar manner the wake fraction for a spherical shape may be obtained directly from (87) when the limit $h \rightarrow_{0}$ is applied. The resulting expression is

$$
\bar{W}_{e}=\left(\frac{a_{1}}{x_{p}}\right)^{3}\left[1-\left(a_{1 / x_{p}}\right)^{3}\right]\left\{1+\frac{A}{4 \pi x_{p}^{2}}\left[\sqrt{1+\bar{c}_{T+1}}-1\right]\right.
$$

which can be also obtained by applying the Lagally theorem to a sphere represented by an $\mathrm{x}$-oriented doublet at the origin. 


\section{REFERENCES}

1. Byrd, P.F., and Friedman, M.D., "Handbook of Elliptic Integrals", 2nd ed., Springer Verlag, New York, (1971).

2. Cummins, W.E., "The Forces and Moments Acting on Body Moving in Arbitrary Potential Stream", J. Ship Research, Vol. 1, No. 1, pp. 7-18, (1957).

3. Dickmann, H.E., "Thrust Deduction, Wave Resistance of a Propeller and Interaction with Ship Waves, 'Ingenieur Archiv, Vol. 9, pp. 452-486, (1938).

4. Eggers, K.W.H., "Über Singularitätendarstellungen und Kraftwirkungen bewegter Körper in imkompressibler F1üssigkeit", Schiffstechnik, Vo1. 38, pp. 3-15, (1960).

5. Hobson, E., "The Theory of Spherical and E1lipsoidal Harmonics", Chelsea, New York, (1955).

6. Kochin, N.E., Kibel, I.A., and Roze, N.V., "Theoretical Hydrodynamics", Interscience Publishers, John Wiley and Sons, (1964).

7. Korvin-Kroukovsky, B.V., "Stern Propeller Interaction with Streamline Body of Revolution", International Shipbuilding Progress, Vol. 3, pp. 3-24, (1956).

8. Lagally, M., "Berechnung der Kråfte und Momente die Stromende Flüsigkeiten auf ihre Begrenzung Ausüben", Z. Angew Math Mech. Vo1. 2, No.6, pp. 409-422, (1922).

9. Lamb, H., "Hydrodynamics", Dover, New York, (1932).

10. Landweber, L., and Yih, C.S., "Forces, Moments and Added Masses for Rankine Bodies", J. Fluid. Mech, Vo1. 1, Part 3, pp. 319-336, (1956).

11. Martinek, J., and Yeh, G.C.K., "On Potential Wake and Thrust Deduction", International Shipbuilding Progress", Vo1. 1, pp. 79-82, (1954).

12. Miloh, T., "Forces and Moments on a Tri-Axial Ellipsoid in Potential Flow", Israel J. of Tech., Vol. 11, Nos. 1-2, pp. 63-74, (1973).

13. Miloh, T., "The U1timate Image Singularities for External E1lipsoidal Harmonics", S.I.A.M. J. of App1. Math., Vo1. 26, No. 2, pp. 334-344, (1974).

14. Miloh, T., and Landweber, L., "Ship Centerplane Source Distribution", in preparation.

15. Newman, J.N., "Water Wave Singularities in a Prolate Spheroidal Coordinate System", J. Ship. Research, Vo1. 14, pp. 41-46, (1972).

16. Nowaki, H., and Sharma, S.D., "Free Surface Effects in Hu11-Propeller Interaction", Dept. of Naval Architecture and Marine Engineering, University of Michigan, Rep. No. 112, (1971). 
17. Taylor, G.I., "The Forces on a Body Placed in a Curved or Converging Stream of Fluid", Proc. Roy. Soc., Ser. A., No. 120, (1928).

18. Tsakonas, S, and Jacobs, W.R., "Potential and Viscous Parts of the Thrust Deduction and Wake Fraction for an Ellipsoid of Revolution", J. of Ship Research, Vol. 4, pp. 1-16, (1960).

19. Walter, H.G., "Lame Functions of the.First Kind Generated by Computer", Celestial Mechanics, Vol. 4, pp. 15-30, (1971).

20. Weinblum, G., "Rotationskörper geringsten Wellenwiderstandes", Ingenieur Archiv., Vol. 7, pp. 104-117, (1936).

21. Whittaker, E.T., and Watson, G.N.", A Course of Modern Analysis", Cambridge University Press, 4th ed., (1935).

22. Wu, T.Y., and Chwang, A.T., "Double-Model Flow Theory - A New Look at a Classical Problem", Proceedings of the Tenth ONR Symposium on Naval Hydrodynamics at M.I.T., (1974). 